\title{
An Instrumented Manipulandum For Human Grasping Studies
}

\author{
Alessandro Altobelli ${ }^{1}$, Matteo Bianchi ${ }^{1}$, Manuel G. Catalano ${ }^{1}$, Alessandro Serio ${ }^{2}$, \\ Gabriel Baud-Bovy ${ }^{3}$, and Antonio Bicchi ${ }^{1}$
}

\begin{abstract}
This work presents a novel haptic device to study human grasp, which integrates different technological solutions thus enabling, for the first time, to achieve: (i) a complete grasp characterization in terms of contact forces and moments; (ii) an estimation of contact point location for varying-orientation contact surfaces; (iii) a compensation of force/torque offsets and estimation of the mass and center of mass of the device, for different orientations and configurations in the workspace; (iv) different stiffness properties for the contact points, i.e. rigid, compliant non-deformable and compliant deformable, thus allowing to study the effects of cutaneous cues in multi-finger grasps. In addition, given the modularity of the architecture and the simple mechanism to attach/detach the contact modules, this structure can be easily modified in order to analyze different multi-finger grasp configurations. The effectiveness of this device was experimentally demonstrated and applications to neuroscientific studies and state of the art of devices for similar investigations are discussed in depth within the text.
\end{abstract}

\section{INTRODUCTION}

Understanding human hand organization and control is a fundamental step to build robotic hands with comparable performance. Although merely bio-mimicking human behaviour is clearly unfeasible (and senseless), observations made in human hands can provide inspiration to robotics, if properly translated into a language understandable by an artificial body [1]. This motivates the high number of studies on human grasp and manipulation (e.g. [2], [3], [4]), which require accurate measurements of forces and torques at contact as well as contact point estimation to achieve a complete mathematical description and comprehension of these phenomena.

Force-related measurements can be obtained with different degrees of completeness and precision. On the one side, sensors can be directly fixed on the hand and worn by users as gloves to enhance versatility [5]. However such gloves, which are usually composed of pressure sensors, can only measure the normal contact force and interferences on hand motion can also occur. In [6] shear force measurements were also achieved through a fingernail sensor and exploiting the correlation between blood distribution under the finger nail and forces. However, information on the contact points

${ }^{1}$ A. Altobelli, M. Bianchi, M. G. Catalano and A. Bicchi are with Department of Advanced Robotics (ADVR), Istituto Italiano di Teconlogia, 30 Via Morego, 16163 Genova, Italy and with Centro di Ricerca "E. Piaggio", Università di Pisa, 1 Largo L. Lazzarino, 56122 Pisa, Italy. $\{a$. altobelli, m. bianchi, m.catalano\} at iit.it, bicchi at centropiaggio.unipi.it

${ }^{2}$ A. Serio, is with Centro di Ricerca "E. Piaggio", Università di Pisa, 1 Largo L. Lazzarino, 56122 Pisa, Italy. a.serio at centropiaggio.unipi.it

${ }^{3} \mathrm{G}$. Baud-Bovy is with the Department of Robotics, Brain and Cognitive Systems (RBCS), Istituto Italiano di Tecnologia, 30 Via Morego, 16163 Genova, Italy. Gabriel. Boud-Bovy at it.it

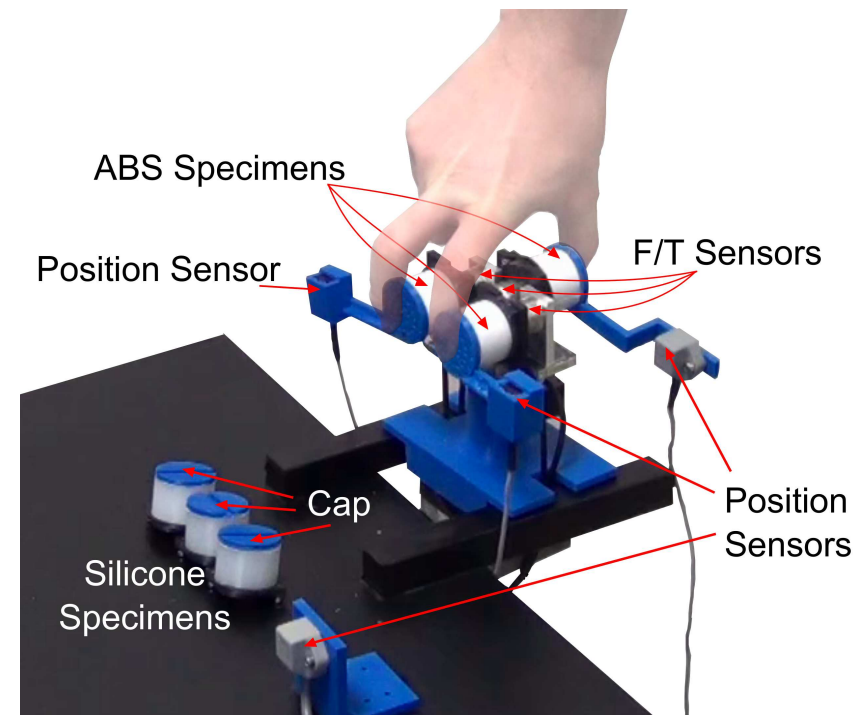

Fig. 1: The manipulandum with its main features.

still lacks. A recent promising solution was provided by ThimbleSense [7], an individual-digit wearable tactile sensor that can measure all the wrench components together with contact point location. Nonetheless, wearing gloves or fixing sensors on the fingertips prevents a direct contact of the fingerpad with the external objects, impeding a proper stimulation of cutaneous sensors that play an important role in object manipulation tasks and grip control [8].

On the other side, it is possible to directly sensorize objects or devices [3] by mounting force/torque (F/T) and/or tactile sensors on a rigid structure. One of the limitations of this approach is that it is difficult to change the shape of the object. To overcome this limitation, the structure of the device is sometimes built so that the orientations of the contact surfaces can be varied (e.g. [9]). Another limitation is that not all components of the external wrench or contact locations can be measured. In [10] these limitations are partially overcome using a modular multi degrees of freedom (DoFs) F/T sensor, which was composed of six 6-axis F/T sensors spatially organized on the face of a cube, within a sensorized object capable of multi-touch detection.

At the present time, the best approach to measure physical interaction with the object, as well as the degree of precision and completeness needed, depends on the aspect of human grasp that is under investigation. The two aforementioned approaches (hereinafter also referred to as "humanside" and "object-side", respectively) exhibit pros and cons and it is difficult (if not impossible) to design a system that can fully measure the physical interaction that occurs be- 
tween the hand and the object during arbitrary manipulation tasks. In this respect, it is noteworthy that almost all studies on multi-digit grasp have focused on the control of finger forces during the manipulation of rigid objects (e.g. [2], [3], [9]). To our knowledge, there are only a few studies that have investigated how humans control contact forces in multi-finger grasping of deformable or soft objects, despite the fact that hardness/softness is an important characteristic of objects [11] and one of the first haptic cues that infants can use to discriminate objects and squeeze them in their hands [12]. Furthermore, the constraints and force control strategies involved in manipulating fragile or deformable objects might differ from those involved in the manipulation of rigid objects. For example, avoiding large contact forces might be crucial to avoid deforming or breaking them. The grasp might also be more or less stable depending on the properties of the object.

The effect of compliance when holding an object with the tripod grasp was investigated in [13] with a device, where a spring was placed below each contact. The control of the contact force when holding a fragile objects with a prismatic grasp was investigated in [14], with a device that collapsed when the contact force exceeds some thresholds.

To further investigate these issues, in a previous work ([15]) we realized a device that enabled to vary the stiffness of contact points in an independent and controllable fashion. Unlike [13], this device allowed the experimenter to vary the contact point stiffness through haptic softness displays. Six DoF F/T sensors measured the contact forces exerted by participants and the contact point was estimated taking into account the actual position of the contact surface [16]. Although such a device might be profitably used for hand rehabilitation, its usage in human studies can be limited because the cutaneous cues at contact can be impaired by the conical shape of the displays, which can indent the user's fingerpad thus producing a sort of "hooking effect".

Finally, the system, as well as all the other systems reported in the Introduction as a review of the state of the art, is used in a grasp configuration parallel to the ground, to disentangle in different configurations the components due to the weight of the object from the force components exerted at the contacts, and to deal with the offsets that affect F/T measurements. However, this clearly represents a notable restriction of the general case.

In this paper we propose a modular manipulandum to be used in tripod grasp studies (see also fig. 1), where the contacts can be easily changed thanks to a mechanical system. These contacts can be rigid or consist of silicone specimens. The latter ones can be covered with a rigid surface, thus enabling contact point estimation using the algorithms and techniques reported in [16] and the integration with a motion capture system to estimate surface orientation. Indeed, in this case, where the surface can assume different orientations, contact point estimation would be not possible only using F/T measurements.

Without the rigid cap, users can interact with a naturalistically deformable surface, thus enabling a proper usage of cutaneous information for grip control, while F/T quantities were recorded. Furthermore, the ease to change the contact modules and the modularity of the architecture can be

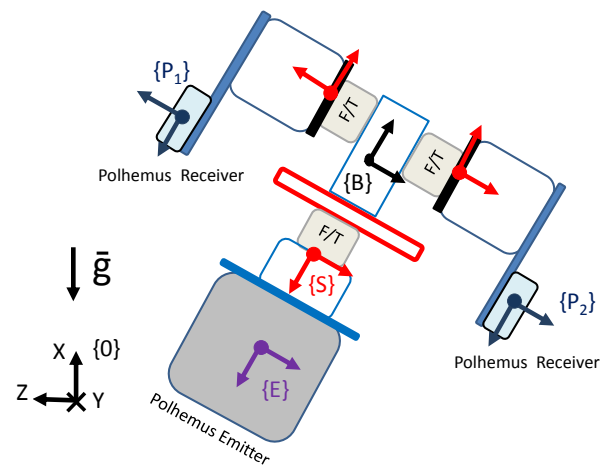

Fig. 2: The manipulandum structure with reference frames and components reported.

used to increase/modify the number of contacts and easily generalized to different object shape.

Additionally, we suitably exploited the techniques reported in [18], [19] to define a procedure to handle F/T sensor offsets and to estimate the inertial parameters of the device (w.r.t. the local frame of each force sensor) in static conditions, i.e. the mass and the coordinates of the center of mass. In this manner the manipulandum can be profitably used for grasping experiments in any arbitrary configuration, since it allows to correctly define contact force/torque and external wrench components and to obtain reliable force measurements. The effectiveness of the here reported techniques is shown for different manipulandum orientations and applied forces.

\section{Mechanical StRUCtURE}

The instrumented manipulandum includes three contact surfaces which can be grasped with a tripod layout. In our experiments, the thumb in opposition to the index and middle finger was used to grasp the manipulandum, palm down. Each contact surface consists of a contact module that can be easily attached/detacched to/from the structure of the manipulandum, through an interface engineered in Acrilonitrile-Butadiene-Stirene (ABS) rapid prototyping material. The structure of the manipulandum was fabricated in aluminium using CNC (Computer Numerical Control) machine to ensure structural rigidity.

Each contact module consists of a cylindrical base in ABS (rigid case, Young Modulus $1.4 \mathrm{GPa}$ ) or silicone. The silicone was obtained by mixing a given quantity of a commercial bicomponent, room temperature-curing silicone (BJB TC-5005A/B), with a percentage of plasticizer (BJB TC-5005C), acting as a softener of $20 \%$. The Young modulus of the silicone is $510 \mathrm{kPa}$ [20]. By changing the percentage of plasticizer, the stiffness of the contact also changes.

The contact modules can come endowed with a rigid cap in ABS, where the receiver of the Polhemus magnetic system $^{1}$ (Colchester, VT -US) is attached trough a rigid arm/support. The emitter is placed on the bottom part of the

\footnotetext{
${ }^{1}$ The static accuracy of the Polhemus system, in terms of Root Mean Square Error (RMS) is $0.03 \mathrm{in} \cong 0.762 \mathrm{~mm}$ for the position and $0.15^{\circ}$ for the orientation
} 


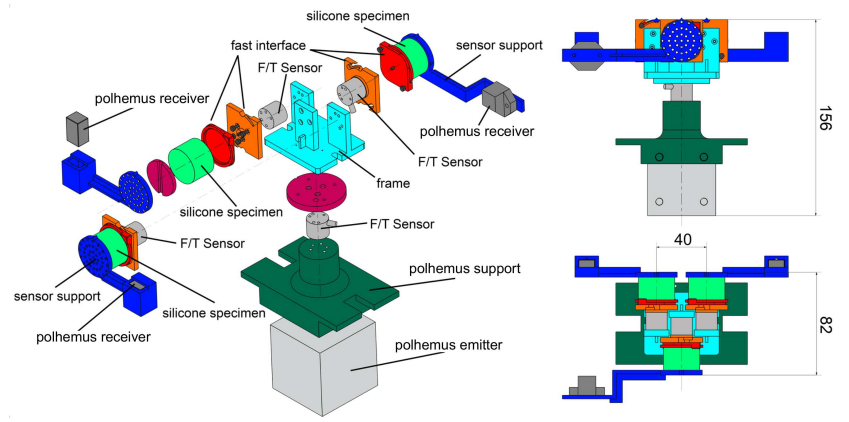

Fig. 3: Exploded drawing view of the manipulandum and its main features with dimensions in [mm].

manipulandum. In this manner, we can measure the position and orientation of the cap surface w.r.t. the emitter frame $\{E\}$. An additional receiver is attached to the table where the manipulandum is placed in rest conditions (see fig. 1). In this manner, an inertial reference frame $\{0\}$ can be defined. For further details please refer to fig. 2. The support frame for the receivers was realized in ABS and the distance from the aluminium frame was heuristically chosen in order to avoid any possible electromagnetic interference. Other possible electromagnetic interferences from external sources were not detected by the Pholemus system within the operating workspace. Three force-torque sensors (Series Nano 17 by ATI, Apex, NC, USA) were positioned below the interface where the contact modules are attached/detached to measure the force and torque components applied by each finger. In this manner we can have three experimental conditions: (i) rigid ( $\mathrm{ABS}$ module, $\mathrm{w}$ or w/o rigid cap); (ii) compliant non-deformable (silicone with rigid cap); (iii) compliant deformable (silicone w/o rigid cap). In condition (iii) we can only estimate F/T components, since the algorithm used for contact point detection in [16] requires information on surface orientation, which can not be achieved without Polhemus system. Future works will be devoted to find a manner to estimate contact surface orientation also in this case, for example through Finite Element (FE) modeling.

A fourth F/T sensor placed at the basis of the structure provides an independent measure of the weight of the manipulandum and external wrench, when the object is lifted. The total weight of the manipulandum, including the sensor cables, is around $540 \mathrm{~g}$, but it might be easily varied with additional external loads which could be added to the base of the device. An exploded drawing view of the device with dimensions is reported in fig. 3.

All the systems are integrated and synchronized in Simulink (Matlab R2012a) with Simulink Block for Real Time Execution and each acquisition is performed at 100 $\mathrm{Hz}$.

\section{Contact Point Estimation}

In order to estimate fingertip contact points on the tripod during grasp tasks, we use the Intrinsic Tactile Sensing Algorithm (ITSA, for more details see also [10] and [16]). Briefly, the ITSA can compute contact points from F/T measurements and from the knowledge of the shape equation

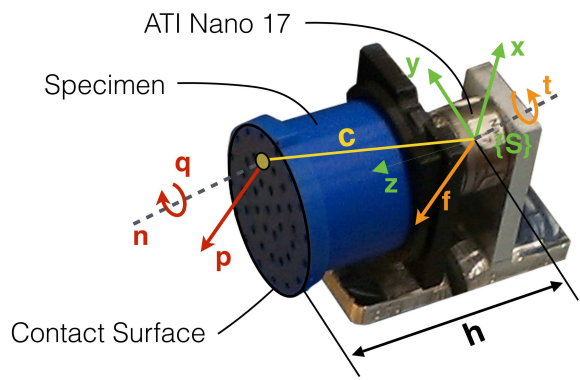

Fig. 4: Application of the Intrinsic Tactile Sensing Algorithm (ITSA) on a contact surface of the tripod. Main features for the contact point detection are highlighted.

of the surfaces fixed on the F/T sensor (see fig. 4). For the sake of clarity, here, we recall the ITSA for a single tactile surface of the tripod considering that the algorithm can be easily replicated for all the contact surfaces. The position of the contact point ${ }^{S} c \in \mathbb{R}^{3}$ (i.e. with components $\left.\left[{ }^{S} c_{x},{ }^{S} c_{y},{ }^{S} c_{z}\right]^{T}\right)$ is expressed w.r.t. force sensor reference frame $\{S\}$. Let $h \in \mathbb{R}^{3}$ (with components $\left[h_{x}, h_{y}, h_{z}\right]^{T}$ ) be the offset of the surface fixed on the F/T sensor, achievable via Polhemus measurements. We can obtain the position of the contact point as

$$
s_{c}=\frac{\left({ }^{S} \bar{f} \times{ }^{S} t+h\left\|^{S} \bar{f}\right\|^{S} f\right)}{\left\|{ }^{S} \bar{f}\right\|^{2}}
$$

where ${ }^{S} f \in \mathbb{R}^{3}$ and ${ }^{S} t \in \mathbb{R}^{3}$ are the contact force and torque measured by the force-torque sensor while ${ }^{S} \bar{f}=\left[0,0,{ }^{S} f_{z}\right]^{T}$. Coherently all the contact points are on the planar surface available to touch. It is possible to notice that the ITSA not only detects the contact point but also computes its related forces $p \in \mathbb{R}^{3}$, torques $q \in \mathbb{R}^{3}$ and contact normal $n \in \mathbb{R}^{3}$ (see also fig. 4 for more details). For measurement homogeneity, for each contact surface, after the application of the ITSA and, thus, the detection of the contact point, we transport sensor frame $\{S\}$ into the inertial frame $\{0\}$.

\section{F/T COMPENSATION}

In order to achieve reliable $\mathrm{F} / \mathrm{T}$ measurements, the sensors are zeroed in a known configuration before each acquisition. However, when the manipulandum is arbitrarily placed in space, it is important to estimate the mass and the center of mass coordinates (the latter ones are not invariant w.r.t. translation and rotation) of the structure attached to the sensor, to enable a correct offset compensation. To properly handle these problems, we suitably implemented and applied the techniques described in [18], [19]. To do this, we collected F/T measurements in a large number of manipulandum configurations (larger than 3000) in the workspace, while the reference system of the sensors $\{S\}$ (whose position w.r.t. the receiver sensor is known) can be computed w.r.t. the inertial one from the Polhemus measurements.

For the fourth sensor, the estimation procedure will lead to the identification of the components of the external 
wrench that will be used in the grasp equation. In our case, this estimation is conducted in static conditions, i.e. the inertial parameters are zero, except for the mass and the center of mass of the part of the manipulandum attached to the sensor. Without loss of generality, we report the procedure only for the fourth sensor. The parameters to be estimated are: the mass of the object attached to the sensor $m$, the center of mass coordinates $\left(\left[M_{x}, M_{y}, M_{z}\right] \in \mathbb{R}^{3}\right)$ expressed in the sensor frame $\{S\}$ and the offset vector components (of force and torque) w.r.t. $\{S\}$, i.e. $\left[f_{B}, \tau_{B}\right] \in \mathbb{R}^{6}$. The $\mathrm{F} / \mathrm{T}$ readings are collected for each i-th sample in the external wrench vector defined as $w_{i}=\left[f_{x}, f_{y}, f_{z}, \tau_{x}, \tau_{y}, \tau_{z}\right]^{T}$ w.r.t. $\{S\}$, while the vector composed with all the unknowns is $\phi=\left[\begin{array}{llllll}m & M_{x} & M_{y} & M_{z} & f_{B} & \tau_{B}\end{array}\right]^{T}$ w.r.t $\{S\}$. In this manner we can write

$$
w_{i}=\left[\begin{array}{cc|c}
\left({ }^{S} R_{0}\right)(-\bar{g}) & 0^{3 \times 3} & I^{6 \times 6} \\
0^{3 \times 1} & \wedge\left(\left({ }^{S} R_{0}\right)(\bar{g})\right) & \phi
\end{array}\right]
$$

where $\bar{g}$ is the gravity vector w.r.t the inertial reference frame $\{0\},{ }^{S} R_{0}$ is the rotation matrix to transport $\{S\}$ into $\{0\}$ and $\wedge(\cdot)$ is the skew-operator. For the i-th wrench recording, we can define

$$
A_{i}=\left[\begin{array}{cc|c}
\left({ }^{0} R_{S}\right)^{-1}(-\bar{g}) & 0 \\
0 & \wedge\left(\left({ }^{S} R_{0}\right)(\bar{g})\right) & I^{6 \times 6}
\end{array}\right]
$$

If we collect $n(n>>1)$ wrench recordings, we can define the linear system

$$
W=\left[\begin{array}{c}
w_{1} \\
w_{2} \\
\vdots \\
w_{n}
\end{array}\right]=\left[\begin{array}{c}
A_{1} \\
A_{2} \\
\vdots \\
A_{n}
\end{array}\right] \phi=A \phi
$$

Then, exploiting the pseudo inverse operator ${ }^{\dagger}$, all the parameters are estimated as: $\phi=A^{\dagger} W$. Once the mass $m$ and the center of mass of the part of the manipulandum attached to the fourth sensor, i.e. above the fourth sensor, are estimated, and hence the offset of F/T measurements, it is possible to individuate the external wrench components w.r.t. $\{0\}$. The offset-corrected $\mathrm{F} / \mathrm{T}$ measurements provided by the sensor can be expressed w.r.t. the frame $\{B\}$, which is placed at the estimated center of mass. The weight in $\{B\}$ can be obtained by multiplying $m$ for the gravity vector $\bar{g}$ (expressed in $\{B\}$ ). Finally the weight and the measured forces can be algebraically summed and expressed in $\{0\}$. In this manner the components of the external wrench in $\{0\}$ can be obtained as well as reliable $\mathrm{F} / \mathrm{T}$ readings in any workspace configuration, as it is shown in fig. 2 .

\section{EXPERIMENTS ON CONTACT POINT ESTIMATION}

To test the accuracy of the contact point detection, we use the ABS contact surface shown in fig. 5a where five aluminium dowel pins (diameter $2 \mathrm{~mm}$, height $0.5 \mathrm{~mm}$ ) with known dimensions and positions w.r.t the center of

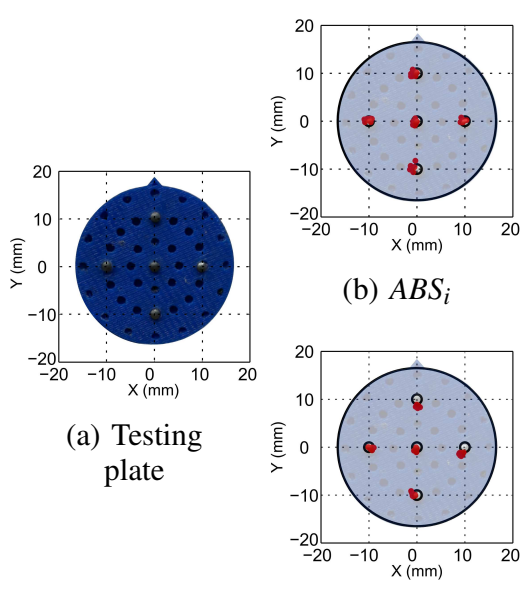

(d) SILICONE

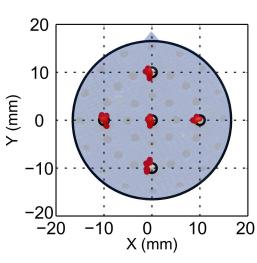

(c) $A B S_{t i}$

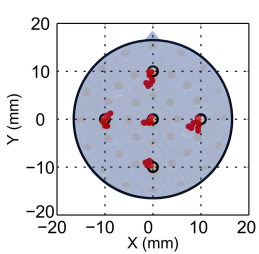

(e) SILICONE $E_{t i}$
Fig. 5: ITSA contact point detection accuracy in different conditions. Red points represent contact point positions estimated by ITSA.

the contact surface were placed. Each spike was touched 10 times with a thin tip and the ITSA was applied after F/T compensation procedure described in Section IV. Four different conditions are considered: $A B S_{i}, A B S_{t i}, S I L I C O N E_{i}$, $S I L I C O N E_{t i}$. The labels $A B S$ and SILICONE indicate the materials of the contacts under the rigid ABS plate where the spikes were placed. The subscript " $i$ " indicates that the manipulandum was tilted w.r.t. the horizontal plane (approximately $30^{\circ}$ ) and normal forces to the surface were applied within the range from 2 to $10 \mathrm{~N}$ and tangential forces were from -1 to $1 \mathrm{~N}$; " $t i$ " indicates that the manipulandum was tilted (approximately $30^{\circ}$ ) and the normal forces were from 2 to $5 \mathrm{~N}$ and the tangential forces were within the range from -3 to $3 \mathrm{~N}$.

\begin{tabular}{ccc}
\hline Tests & Mean Error & Standard Deviation \\
\hline$A B S_{i}$ & $0.24 \mathrm{~mm}$ & $0.20 \mathrm{~mm}$ \\
$A B S_{t i}$ & $0.28 \mathrm{~mm}$ & $0.20 \mathrm{~mm}$ \\
SILICONE $_{i}$ & $0.50 \mathrm{~mm}$ & $0.31 \mathrm{~mm}$ \\
SILICONE $_{t i}$ & $0.90 \mathrm{~mm}$ & $0.65 \mathrm{~mm}$
\end{tabular}

TABLE I: Estimation errors for ITSA. ABS and SILICONE refer to ABS rigid contacts and silicone contacts, respectively.

Estimated contact points are shown in figg. 5b, 5c, 5d, $5 \mathrm{e}$. The error is computed as the absolute difference between the radial distance from the estimated contact point and the center of the spike and the radius of the spike. In Table I, we report the error averaged across all trials and spike locations with related standard deviation for each test type. Even if the silicone case in tilted condition with large tangential forces exhibits the largest mean error, probably due to a non correct coupling between the silicone surface and the $\mathrm{ABS}$, the algorithm for contact point detection with $\mathrm{F} / \mathrm{T}$ compensation still provides satisfactory results (the average error is under $1 \mathrm{~mm}$ ). The results are comparable with those 
reported in [10].

\section{EXPERIMENTS ON GRASPING}

To properly validate the effectiveness of the manipulandum and of the methods here described (contact point detection and $\mathrm{F} / \mathrm{T}$ compensation for different configurations of the device in the workspace), we compute the following equation, which relates the contact force vector $F \in \mathbb{R}^{18}$ to the external wrench $w_{f} \in \mathbb{R}^{6}$ (expressed in $\{0\}$ ), for different manipulandum orientations

$$
w_{f}=G F
$$

where $G$ is the grasp matrix, $\wedge^{\wedge}(\cdot)$ is the skew-matrix operator and $C_{1}, C_{2}$ and $C_{3}$ are the contact point calculated through ITSA and with F/T compensation

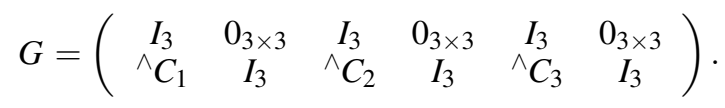

When the object is lifted and held stationary, the external wrench $w_{f}$ corresponds to the weight of the device plus the cables of the sensors.

To validate the model of the manipulandum and the correctness of the force-torque measurements, we checked that the external wrench $w_{f}$ derived from the three forcetorque sensors placed under each finger was equivalent to the external wrench $w_{e}$ measured by the fourth sensor placed in the basis of the manipulandum. Apart from numerical errors, the two estimates should be equal and the following relationship should be verified

$$
w_{e}-w_{f}=w_{e}-G F=0 .
$$

The absolute residual error is computed as : $\left|w_{e}-G F\right|$. We computed the absolute error for 10 different configurations of the manipulandum, with rigid and deformable compliant contacts. The absolute average errors are reported for the two conditions in Table II. In this case, errors are comparable with the one reported in [15] and between the two contact conditions, despite the different orientations of the manipulandum, the uncertainties introduced by the Polhemus and those due to the interface between the contact modules and the cap. These results validate the reliability of the here proposed techniques.

\section{NeURoscientific StUdies AND ApPliCATIONS}

As noted in the Introduction, only a few human studies have investigated the control in multi-digit grasp when holding a deformable objects. The device described here might be used to investigate different motor control issues. One of this issue is whether contact forces are directly controlled by the Central Nervous System (CNS) or if they result from the interaction between central commands from the CNS and biomechanical properties of the human hand. As demonstrated by a large number of recent studies (e.g. [21], [22]), control can be simplified by letting the fingers mold themselves around the object. However, we still know little about how such control occurs in human grasp and the relative importance of force control and passive properties of the hand is highly debated in motor control [23]. In this respect, it is noteworthy that this device provides a way to manipulate the contact compliance under each digit separately, which is crucial to understand interaction between digits [13]. At the same time, the device can provides necessary information about the position of the fingertip (contact point) on the object, which is crucial in the analysis of the grasp. This fact together with the possibility to use the device in any configuration by compensating sensor offset and estimating device inertial parameters can be profitably exploited to investigate models on force control distribution, e.g. equilibrium point [24] and/or virtual finger hypothesis [9], in any arbitrary orientation of the manipulandum, thus enabling a more ecological interaction.

In [17] the device presented in this paper was profitably used in a new set up to study the strategies adopted by humans to modulate the stiffness at fingertips.

Finally, the manipulandum presented in this work also offers the possibility to investigate the contribution of different sensory cues in softness perception, when the silicone cylinders are grasped with and without a rigid cover. It has been suggested that tactile system can provide a direct information about the softness of a deformable object when it is touched with the naked fingertip. In this case, the rate of change of average pressure is invariant with respect to indentation velocity and the object stiffness might be directly encoded in the population response of SAI mechanoreceptors [25]. In contrast, when the deformable object is touched with a rigid probe or when the surface of the compliant object is rigid, it is necessary to integrate proprioceptive and tactile information. For example, stiffness might be estimated from information about the rate of force and indentation velocity provided by tactile and kineasthetic inputs.

\section{CONCLUSIONS AND FUTURE WORK}

In this paper we have presented a modular manipulandum that can be used to study force distribution in human grasp for tripod layouts. The device allows to independently change each of the contact modules, thus varying contact stiffness.

The manipulandum combines $\mathrm{F} / \mathrm{T}$ sensing and motion tracking technology to provide a complete characterization of the contact forces and moments applied on the contact surfaces of an object in any arbitrary orientation. This work represents a great technological effort to integrate different methods and technical solutions, such as (i) the algorithm described in [16] to estimate contact point location for varying-orientation contact surfaces and (ii) the procedures described in [18], [19] to handle force/torque offsets and estimate the mass and the center of mass of the device in different orientations. In addition, given the modularity of the architecture and the simple mechanism used to attach/detach the contact modules, this structure can be easily modified in order to study different multi-finger grasp configurations. In particular, this mechanism can be used to change easily the stiffness properties of the contact surface, thus enabling the study of the effects of cutaneous cues in human grasps. 


\begin{tabular}{ccccccc}
\hline Components & $f_{x}[N]$ & $f_{y}[N]$ & $f_{z}[N]$ & $\tau_{x}[\mathrm{Nmm}]$ & $\tau_{y}[\mathrm{Nmm}]$ & $\tau_{z}[\mathrm{Nmm}]$ \\
\hline ABS & $0.0725 \pm 0.0589$ & $0.0769 \pm 0.0603$ & $0.1150 \pm 0.0738$ & $9.7638 \pm 3.4614$ & $2.8398 \pm 2.2499$ & $3.7975 \pm 2.4095$ \\
SILICONE & $0.0496 \pm 0.0422$ & $0.0470 \pm 0.0408$ & $0.1327 \pm 0.0763$ & $12.2726 \pm 2.5275$ & $1.8448 \pm 1.4165$ & $6.4420 \pm 3.8180$
\end{tabular}

TABLE II: Average absolute residual error with standard deviation for different manipulandum configurations. $A B S$ and SILICONE refer to ABS rigid contacts and silicone contacts, respectively. $f_{x}, f_{y}, f_{z}, \tau_{x}, \tau_{y}, \tau_{z}$ refer to the force and torque components w.r.t. $\{0\}$.

Future works will aim at using this device for neuroscientific studies on human grasp force distribution and control (see Section VII), enabling the investigation of the different factors that influence human grip control, thus advancing the state of the art of "object-side" approaches for force measurements. To combine force measurements with kinematic postural data, integration with portable sensors (e.g. inclinometers) or other motion tracking systems will be also considered. As previously mentioned, additional loads can be added to the base of the manipulandum: future works will investigate grasp force distribution for different object weights. Applications in rehabilitation scenarios will be also evaluated.

\section{ACKNOWLEDGMENT}

This work is supported in part by the European Research Council under the Advanced Grant SoftHands "A Theory of Soft Synergies for a New Generation of Artificial Hands" no. ERC-291166, and by the EU FP7 project (no. 601165), "WEARable HAPtics for Humans and Robots (WEARHAP)".

\section{REFERENCES}

[1] A. Bicchi, M. Gabiccini, and M. Santello, "Modelling natural and artificial hands with synergies," Philosophical Transactions of the Royal Society B: Biological Sciences, vol. 366, no. 1581, pp. 3153 3161, 2011.

[2] J. R. Flanagan, M. K. Burstedt, and R. S. Johansson, "Control of fingertip forces in multidigit manipulation," Journal of Neurophysiology, vol. 81, no. 4, pp. 1706-1717, 1999.

[3] V. M. Zatsiorsky and M. L. Latash, "Multifinger prehension: an overview," Journal of Motor Behavior, vol. 40, no. 5, pp. 446-476, 2008 .

[4] M. Santello, G. Baud-Bovy, and H. Jörntell, "Neural bases of hand synergies," Frontiers in computational neuroscience, vol. 7, 2013.

[5] F. Vecchi, S. Micera, F. Zaccone, M. Carrozza, A. Sabatini, and P. Dario, "A sensorized glove for applications in biomechanics and motor control," in Proceedings of the 2001 Conference of the International FES Society, 2001.

[6] T. R. Grieve, J. M. Hollerbach, and S. A. Mascaro, "Force prediction by fingernail imaging using active appearance models," in World Haptics Conference (WHC), 2013. IEEE, 2013, pp. 181-186.

[7] E. Battaglia, G. Grioli, M. G. Catalano, M. Santello, and A. Bicchi, "Thimblesense: An individual-digit wearable tactile sensor for experimental grasp studies," in Robotics and Automation (ICRA), 2014 IEEE International Conference on, May 2014, pp. 2728-2735.

[8] R. Johansson and G. Westling, "Roles of glabrous skin receptors and sensorimotor memory in automatic control of precision grip when lifting rougher or more slippery objects," Experimental Brain Research, vol. 56, no. 3, pp. 550-564, 1984.

[9] G. Baud-Bovy and J. F. Soechting, "Two virtual fingers in the control of the tripod grasp," Journal of Neurophysiology, vol. 86, no. 2, pp. $604-615,2001$
[10] A. Serio, E. Riccomini, V. Tartaglia, I. Sarakoglou, M. Gabiccini, N. Tsagarakis, and A. Bicchi, "The patched intrinsic tactile object: a tool to investigate human grasps," 2014.

[11] S. J. Lederman and R. L. Klatzky, "Relative availability of surface and object properties during early haptic processing." Journal of Experimental Psychology: Human perception and performance, vol. 23, no. 6, p. 1680, 1997.

[12] P. Rochat, "Mouthing and grasping in neonates: Evidence for the early detection of what hard or soft substances afford for action," Infant Behavior and Development, vol. 10, no. 4, pp. 435-449, 1987.

[13] S. A. Winges, S. E. Eonta, J. F. Soechting, and M. Flanders, "Effects of object compliance on three-digit grasping," Journal of neurophysiology, vol. 101, no. 5, pp. 2447-2458, 2009.

[14] S. L. Gorniak, V. M. Zatsiorsky, and M. L. Latash, "Manipulation of a fragile object," Experimental brain research, vol. 202, no. 2, pp. 413-430, 2010.

[15] A. Altobelli, M. Bianchi, A. Serio, G. Baud-Bovy, M. Gabiccini, and A. Bicchi, "Three-digit grasp haptic device with variable contact stiffness for rehabilitation and human grasping studies," in Control and Automation (MED), 2014 22nd Mediterranean Conference on. IEEE, 2014

[16] A. Bicchi, J. K. Salisbury, and D. L. Brock, "Contact sensing from force measurements," The International Journal of Robotics Research, vol. 12, no. 3, pp. 249-262, 1993.

[17] M. Rossi, A. Altobelli, S. B. Godfrey, A. Ajoudani, and A. Bicchi, "Electromyographic mapping of finger stiffness in tripod grasp: a proof of concept," in Rehabilitation Robotics (ICORR), 2015 IEEE International Conference on.

[18] C. Atkeson, C. An, and J. Hollerbach, "Rigid body load identification for manipulators," in Decision and Control, 1985 24th IEEE Conference on, Dec 1985, pp. 996-1002.

[19] D. Kubus, T. Kroger, and F. Wahl, "On-line rigid object recognition and pose estimation based on inertial parameters," in Intelligent Robots and Systems, 2007. IROS 2007. IEEE/RSJ International Conference on, Oct 2007, pp. 1402-1408.

[20] E. P. Scilingo, M. Bianchi, G. Grioli, and A. Bicchi, "Rendering softness: Integration of kinaesthetic and cutaneous information in a haptic device," Transactions on Haptics, vol. 3, no. 2, pp. 109 - 118, 2010.

[21] M. G. Catalano, G. Grioli, E. Farnioli, A. Serio, C. Piazza, and A. Bicchi, "Adaptive synergies for the design and control of the pisa/iit softhand," The International Journal of Robotics Research, vol. 33, no. 5, pp. 768-782, 2014.

[22] M. Kazemi, J.-S. Valois, J. A. Bagnell, and N. Pollard, "Humaninspired force compliant grasping primitives," Autonomous Robots, vol. 37, no. 2, pp. 209-225, 2014.

[23] D. J. Ostry and A. G. Feldman, "A critical evaluation of the force control hypothesis in motor control," Experimental Brain Research, vol. 153, no. 3, pp. 275-288, 2003.

[24] M. L. Latash, J. Friedman, S. W. Kim, A. G. Feldman, and V. M. Zatsiorsky, "Prehension synergies and control with referent hand configurations," Experimental brain research, vol. 202, no. 1, pp. 213-229, 2010.

[25] M. A. Srinivasan and R. H. LaMotte, "Tactual discrimination of softness: abilities and mechanisms," in Somesthesis and the Neurobiology of the Somatosensory Cortex. Springer, 1996, pp. 123-135. 